\title{
Application of Spatial Analytic Geometry in the Calculation of Adjacent Well Separation Factors
}

\author{
Binbin Diao ,Deli Gao \\ State Key Laboratory of Petroleum Resource and Prospecting, China University of Petroleum, \\ Beijing, 102249, China
}

Keywords: cluster wells, infill well, directional drilling, separation factors, anti-collision.

\begin{abstract}
With the design distance between cluster well and infilling well continuously decreasing, the calculation of separation factor is of great significance in the adjacent well anti-collision problem. This paper contrasts the differences between the currently common pedal curve separation factor and the oriented separation factor and realizes the calculation of these separation factors by using spatial analytic geometry method separation factors, with the separation factor being defined in many ways. The comparative analysis shows that: in general case, the pedal curve separation factor is less than the oriented separation factor; when the reference point and scanning point are in the same spindle direction with the combined error ellipsoid, the former equals to the latter. In directional drilling project of infilling well where the inclinometer data on old well are of low reliability, it is better to consider the pedal curve separation factor as anti-collision basis; However, in the directional drilling project of cluster well where the inclinometer data on old well are of high reliability, it is better to consider the oriented separation factor as anti-collision basis.
\end{abstract}

\section{Introduction}

In recent years, the high-density cluster well and infilling well projects have been gradually carried out in the offshore and onshore oil exploration, and the collision accidents between well being drilled and well drilled have occurred regularly. However, once the penetration through wells drilled resulting from the collision happens, it will cause severe economic losses. At the beginning, the adjacent well distance scan calculations [1-5] is used to evaluate the degree of compliance between actual wellbore trajectory and designed wellbore trajectory and the collision risk between adjacent wells. In 1991, Thorogood J L put forward the separation factor method to assess the collision risk between adjacent wells[6]. In 1996, Brooks A G introduced a method to evaluate the collision possibility between adjacent wells by probabilistic method[7]. For the past few years, the foreign drilling circles have proposed many explanations for separation factors, among which the one obtained by pedal curve method has been widely applied[8]. In 2009, Benny Poedjono raised the new concept of oriented separation factor (OSF) and presented method for calculating the oriented separation factor [9-11].

Both the pedal curve separation factor and oriented separation factor combine the calculations of adjacent well distance scanning and wellbore trajectory error, and adopt the method of combined error ellipsoid during calculation. Compared to the traditional calculations of adjacent well distance scanning and separation factors, they can provide more scientific evidence for inter-well anti-collision decision-making. This paper contrasts the differences between the currently common oriented separation factor and separation factor and conducts preliminary study on spatial analytic geometry algorithm of the separation factors, with these separation factors having their own characteristics however. 


\section{Definitions of Pedal Curve Separation Factor and Oriented Separation Factor}

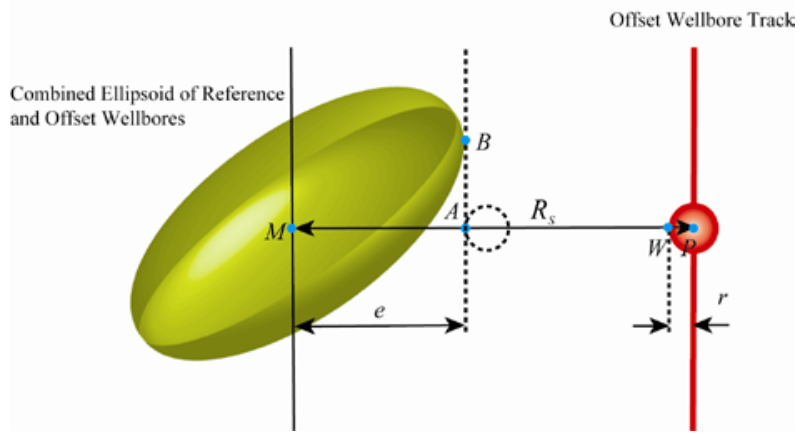

Fig. 1 Diagrammatic drawing of the calculation of pedal curve separation factor

The adjacent well separation factor generally indicates the separation distance between reference point of the reference well and scanning point of the comparison well divides the total errors; The separation distance refers to that scanning radius from the reference point of the reference well to scanning point of the comparison well minuses the well-hole radius sum of reference well and comparison well. Since the concept of adjacent well separation factor was raised, there have been multiple interpretations for the calculation equations of separation factor.

As shown in Fig. 1, point $\mathrm{M}$ refers to the reference point; point $\mathrm{P}$ refers to the scanning point; the line $\mathrm{AB}$ is tangent to the combined error ellipsoid, with the point of tangency being $\mathrm{B}$; the line $\mathrm{AB}$ is perpendicular to the line MP, with the point of intersection being A. At present, the calculation equation for adjacent well separation factor fS used in many commercial softwares can be expressed as[8]:

$$
f_{c s}=\frac{R_{S}-r}{e}
$$

In the equation, Rs refers to scanning radius for the closest distance; $r$ refers to sum of the wellbore radiuses of reference well and comparison well; e refers to the distance between point $\mathrm{M}$ and point A. The separation factor calculated by Eq. (1) can be called as pedal curve separation factor. As seen in Fig. 1, with the reference well and comparison well getting closer, when the point $\mathrm{M}$ and point $\mathrm{A}$ coincide, the pedal curve separation factor equals to 1 and the combined error ellipsoid fails to intersect with the circle of radius $r$. Therefore, when the reference point and scanning point are not in the same spindle direction with the combined error ellipsoid, the adjacent well separation factor overestimates the collision probability of reference well and comparison well.

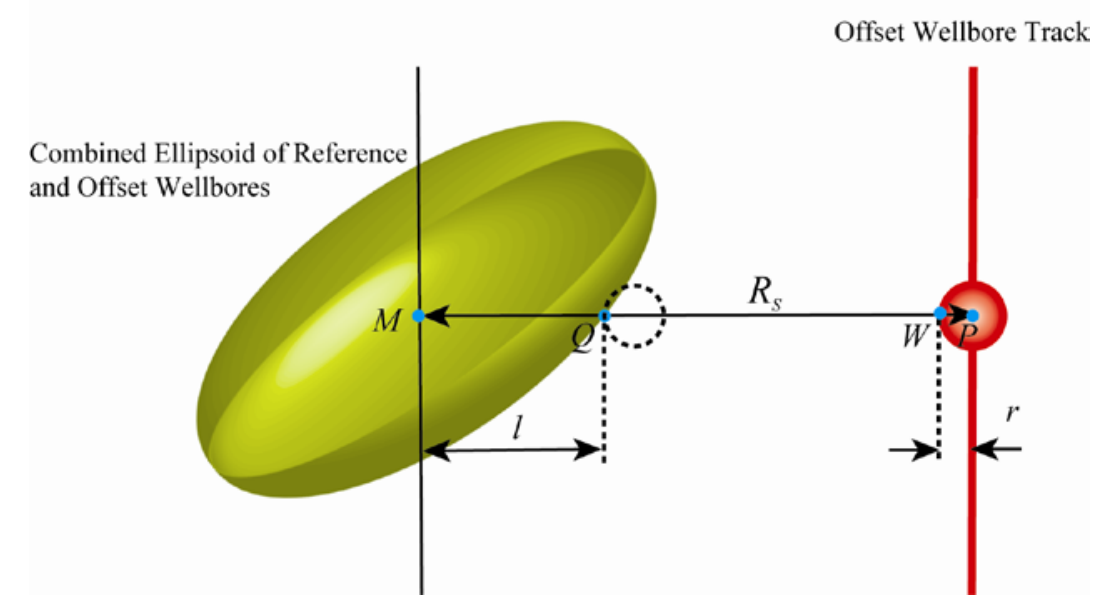

Fig.2 Diagrammatic drawing of the calculation of oriented separation factor

As shown in Fig. 2, point $M$ refers to the reference point; point $\mathrm{P}$ refers to the scanning point; point Q indicates the point of intersection between combined error ellipsoid and scanning radius at reference point and scanning point. According to the definitions of oriented separation factor from Benny Poedjono, the oriented separation factor fos can be expressed as: 


$$
f_{o s}=\frac{R_{S}-r}{l}
$$

Where 1 refers to the distance between point $\mathrm{Q}$ and point $\mathrm{M}$. As seen in Fig. 2, with the reference well and the comparison well getting closer, when the point $\mathrm{M}$ and point $\mathrm{A}$ coincide, the adjacent well oriented separation factor equals to 1 and the combined error ellipsoid is intersected with the circle of radius $r$. Therefore, when the reference point and scanning point are not in the same spindle direction with the combined error ellipsoid, the oriented separation factor underestimates the collision probability of reference well and comparison well.

\section{Calculation of the Pedal Curve Separation Factor}

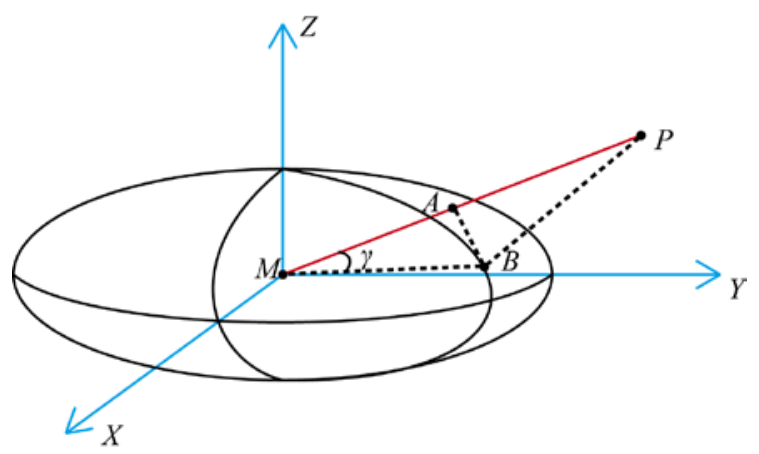

Fig.3 Calculation model of the pedal curve separation factor

The lengths of three semi-major axes (a, b and c) of the combined error ellipsoid and the coordinates (XP, YP, ZP) of reference point $\mathrm{P}$ in the principal axis coordinate system of the ellipsoid with the scanning point $\mathrm{M}$ being the origin of coordinates can be obtained on basis of the methods introduced in the paper [12]. Suppose the coordinates of point of tangency B is (XB, YB, $\mathrm{ZB}$ ) in coordinate system MXYZ and then:

$$
\frac{X_{B}^{2}}{a^{2}}+\frac{Y_{B}^{2}}{b^{2}}+\frac{Z_{B}^{2}}{c^{2}}=1
$$

The plane $\Pi$ where the cross-point $B$ is perpendicular to line MP can be expressed as:

$$
X_{P}\left(x-X_{B}\right)+Y_{P}\left(y-Y_{B}\right)+Z_{P}\left(z-Z_{B}\right)=0
$$

As the plane $\Pi$ is the tangent plane of combined error ellipsoid, it can also be expressed as:

$$
\frac{2 X_{B}}{a^{2}}\left(x-X_{B}\right)+\frac{2 Y_{B}}{b^{2}}\left(y-Y_{B}\right)+\frac{2 Z_{B}}{c^{2}}\left(z-Z_{B}\right)=0
$$

From Eqs. (4) and (5), it can be obtained that:

$$
\left\{\begin{array}{c}
X_{B}=k a^{2} X_{P} \\
Y_{B}=k b^{2} Y_{P} \\
Z_{B}=k c^{2} Z_{P}
\end{array}\right.
$$

Where $\mathrm{k}$ is the intermediate variable. Substitute Eq. (6) into Eq. (3) and then:

$$
k= \pm\left(\frac{1}{a^{2} X_{P}^{2}+b^{2} Y_{P}^{2}+c^{2} Z_{P}^{2}}\right)^{\frac{1}{2}}
$$

On account that point $\mathrm{P}$ and point $\mathrm{B}$ are in the same quadrant of coordinate system MXYZ, so

$$
k=\left(\frac{1}{a^{2} X_{P}^{2}+b^{2} Y_{P}^{2}+c^{2} Z_{P}^{2}}\right)^{\frac{1}{2}}
$$

The coordinates of point of tangency B can be worked out with Eqs. (6) and (8) and then the cosine value of angle $\gamma$ can be calculated:

$$
\cos \gamma=\frac{|M P|^{2}+|B M|^{2}-|B P|^{2}}{2|M P||B M|}
$$

Where $|\mathrm{MP}|$ and $|\mathrm{BM}|$ refer to the distances from point $\mathrm{M}$ to point $\mathrm{P}$ and point $\mathrm{B}$ respectively; $|\mathrm{BP}|$ refers to the distance from point $\mathrm{B}$ to point $\mathrm{P}$. On account that the line $\mathrm{AB}$ is perpendicular to 
line MP, the distance from point $\mathrm{A}$ to point $\mathrm{M}$ can be obtained by the equation below:

$$
e=|A M|=|B M| \cos \gamma
$$

Substitute the obtained e into Eq. (1), and combine adjacent well distance scanning calculations to compute the scanning radius RS and then the value of pedal curve separation factor.

\section{Calculation of the Oriented Separation Factor}

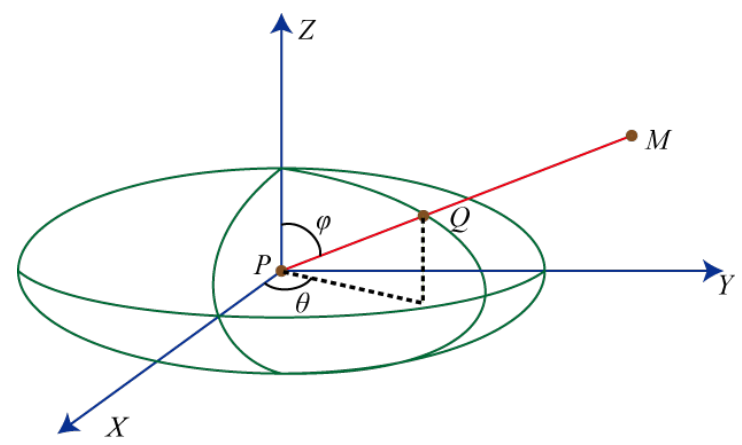

Fig. 4 Calculation model of the oriented separation factor

As shown in Fig. 4, the reference point $\mathrm{P}$, scanning point $\mathrm{M}$ and the point of intersection $\mathrm{Q}$ of scanning radius and combined error ellipsoid are on a straight line, therefore

$$
\left\{\begin{array}{l}
\sin \theta=\frac{Y_{P}}{\sqrt{X_{P}+Y_{P}}} \\
\cos \theta=\frac{X_{P}}{\sqrt{X_{P}+Y_{P}}} \\
\sin \varphi=\frac{\sqrt{X_{P}+Y_{P}}}{R_{S}} \\
\cos \varphi=\frac{Z_{P}}{R_{S}}
\end{array}\right.
$$

Where $\varphi$ refers to the angle between $\mathrm{Z}$ axis and the scanning radius; $\theta$ refers to the angle between $\mathrm{X}$ axis and the scanning radius projected onto the plane PXY. From the parameter equation of ellipsoid, the coordinates (XQ, YQ, ZQ) of point of intersection Q can be expressed as:

$$
\left\{\begin{array}{l}
X_{Q}=a \cos \theta \sin \varphi \\
Y_{Q}=b \sin \theta \sin \varphi \\
Z_{Q}=c \cos \varphi
\end{array}\right.
$$

Due to that the distance between point of intersection $\mathrm{Q}$ and scanning point $\mathrm{M}$ can be expressed as:

$$
l=\sqrt{X_{Q}^{2}+Y_{Q}^{2}+Z_{Q}^{2}}
$$

Therefore, as long as the minimum confidence factor is given, the distance l between point of intersection $\mathrm{Q}$ and scanning point $\mathrm{M}$ can be acquired. Then substitute $\mathrm{l}$ into Eq. (2) to get the oriented separation factor. 


\section{Contrastive Analysis}

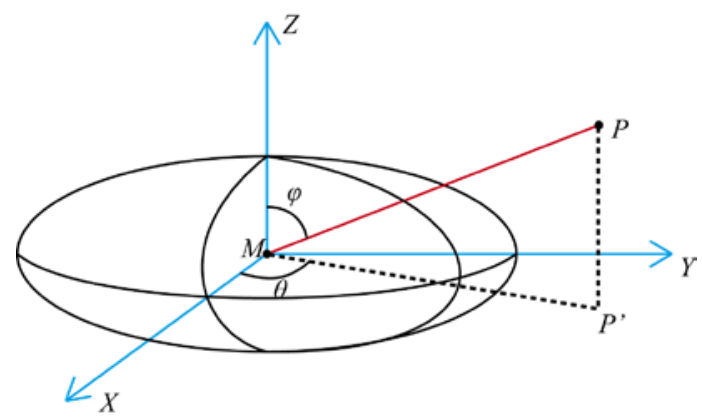

Fig. 5 The relative position between scanning point and combined error ellipsoid

As shown in Fig. 4, point $P$ ' refers to the projection of scanning point $P$ onto the plane $M X Y$; $\theta$ refers to the angle between line $M P^{\prime}$ and $X$ axis; $\varphi$ refers to the angle between line $M P$ and $Z$ axis. Suppose the lengths of three semi-major axes $(a, b$ and $c$ ) of the combined error ellipsoid are $10 \mathrm{~m}$, $4 \mathrm{~m}$ and $1.5 \mathrm{~m}$ respectively, the distance between reference point $M$ and scanning point $P$ is $15 \mathrm{~m}$ and the wellbore radiuses at reference point and scanning point are $241.3 \mathrm{~mm}$. When the angle separately equals to $0, \pi / 6, \pi / 3$ and $\pi / 2$, the variation curves with the angle $\varphi(0 \leq \varphi \leq \pi)$ of pedal curve separation factor and oriented separation factor are shown in Fig. 5. In view of the ellipsoidal symmetry and as seen in the Fig. 5, when the reference point and the scanning point are in the same spindle direction with the combined error ellipsoid, the pedal curve separation factor equals to the oriented separation factor; In other cases, the former is less than the latter. Therefore, compared to the oriented separation factor, it is safer to make the pedal curve separation factor as the anti-collision basis for directional drilling engineer in design and construction of the directional well.
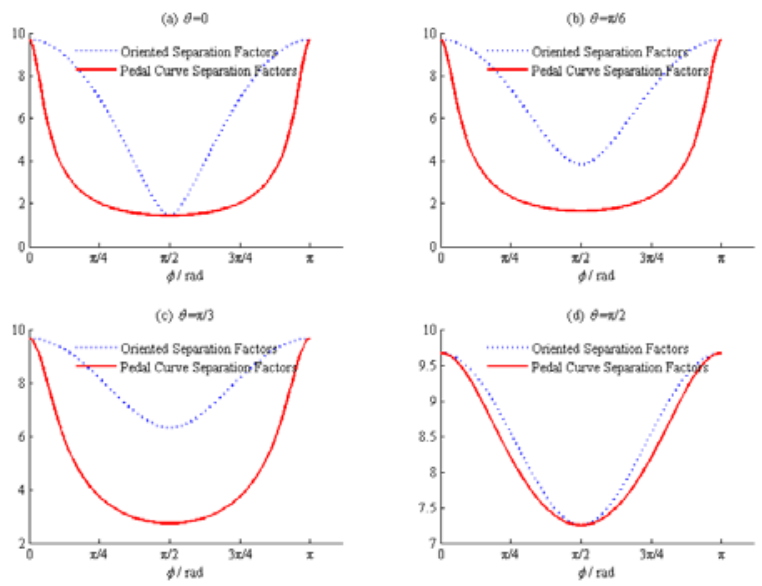

Fig. 5 The variation curve with angle $\varphi$ of pedal curve separation factor and oriented separation factor when the angle $\varphi$ takes different values

\section{Conclusions}

(1) With the design distance between cluster well and infilling well decreasing, the calculation of separation factors is of great significance in the design of cluster well and infilling well and the construction of directional drilling.

(2) The spatial analytic geometry method can be used to achieve the rapid calculation of pedal curve separation factor and oriented separation factor.

(3) When the reference point and the scanning point are in the same spindle direction with the combined error ellipsoid, the pedal curve separation factor equals to the oriented separation factor; In other cases, the former is less than the latter.

(4) Though the pedal curve separation factor and the oriented separation factor have been widely applied on drilling site, these separation factors still present their own disadvantages, therefore a new separation factor is required to put forward to reflect the collision risk between adjacent wells 
in a more scientific manner.

\section{Acknowledgements}

The authors gratefully acknowledge the financial support of the Natural Science Foundation of China (NSFC, 51221003, 51304221). This research was also supported by the other projects (Grant numbers: 2011ZX05009).

\section{References}

[1] Han Zhiyong, Ning Xiuxu. Principle of computer plotting of adjacent well minimum distance scanning diagram. Petroleum Drilling Techniques,1990, 18(1): 1-3.

[2] Gao Deli,Han Zhidong.Principle of computer plotting and calculations of adjacent well distance scanning. Oil Drilling \& Production Technology,1993,15(5): 21-29.

[3] Liu Xiushan; Cen Zhangzhi. Description and calculation of relative positions of wellbore trajectories. Oil Drilling \& Production Technology,1999, 22(3): 7-12.

[4] Lu Gang,Xing Yude,Wu Junlin,et al. Fast scanning algorithm of the adjacent well anti-collision calculations. Petroleum Geology and Engineering, 2007, 21(2): 78-81.

[5] Lu Gang, Chang Hanzhang, Xing Yude, et al. Fast algorithm for scanning the nearest distance among adjacent wells. Petroleum Drilling Techniques, 2007, 35(3): 23-26.

[6] Thorogood J L, Hogg T W and Williamson H S. Application of risk analysis methods to subsurface well collisions. SPE 23941, 1991.

[7] Brooks A G and Wilson H. An improve method for computing wellbore position uncertainty and its application to collision and target intersection probability analysis. SPE 36863, 1996.

[8] McNair G A, Lance S J, Codling J et al. Implementation of a new risk based well collision avoidance method. SPE 92554, 2005.

[9] Poedjono B, Conran G, Akinniranye G et al. Minimizing the risk of well collisions in land and offshore drilling. SPE 108279, 2007.

[10] Poedjono B, Van C P, Lombardo G J et al. Anti-collision risk management for real-world well placement. SPE 121094, 2009.

[11] Poedjono B, Lombardo G J and Phillips W. Anti-collision risk management standard for well placement. SPE 121040, 2009.

[12] Diao Binbin, Gao Deli. Calculation method of adjacent well oriented separation factors. Petroleum Drilling Techniques, 2012, 40(1): 22-27. 